\title{
Optical coherence tomography using nonlinear optics in fiber for broadband source generation
}

\author{
I-Jen Hsu ${ }^{\mathrm{a}}$, Chih-Wei Lu ${ }^{\mathrm{a}}$, Chih-Ren Deng ${ }^{\mathrm{a}}$, C.C. Yang ${ }^{\mathrm{a}, *}$, Chun-Pin Chiang ${ }^{\mathrm{b}}$, \\ Chii-Wann Lin ${ }^{c}$, Yean-Woei Kiang ${ }^{\mathrm{d}}$ \\ a Department of Electrical Engineering and Graduate Institute of Electro-Optical Engineering, National Taiwan University, \\ 1, Roosevelt Road, Sec. 4, Taipei, Taiwan, ROC \\ b Department of Dentistry, National Taiwan University, Taipei, Taiwan, ROC \\ ${ }^{\mathrm{c}}$ Graduate Institute of Bioengineering, National Taiwan University, Taipei, Taiwan, ROC \\ ${ }^{\mathrm{d}}$ Department of Electrical Engineering and Graduate Institute of Communication Engineering, \\ National Taiwan University, Taipei, Taiwan, ROC
}

Received 6 November 2001; received in revised form 9 July 2002; accepted 4 September 2002

\begin{abstract}
An optical coherence tomography (OCT) system of sub-10- $\mu \mathrm{m}$ resolution, with its broadband source generated through nonlinear optics processes of a femtosecond Ti:sapphire laser in a single-mode fiber, was built for biological tissue scanning. With $400 \mathrm{~mW}$ power coupled into the fiber, a spectral full-width-at-half-maximum (FWHM) of $46 \mathrm{~nm}$, centered at $840 \mathrm{~nm}$, could be obtained. The available power for sample illumination could be as high as $100 \mathrm{~mW}$. This condition was used to achieve the OCT depth resolution of $8.6 \mu \mathrm{m}$ in tissues. The OCT system was used for scanning a human gingival mucosa sample. Cavity structures were observed that were quite consistent with the results of histological examination.
\end{abstract}

(c) 2002 Published by Elsevier Science B.V.

Keywords: Optical coherence tomography; Broadband source; Nonlinear optics; Oral cancer

\section{Introduction}

Optical coherence tomography (OCT) and optical Doppler tomography (ODT) have been widely studied for high-resolution subsurface tissue scanning [1-5]. They were developed for di-

\footnotetext{
${ }^{*}$ Corresponding author. Tel.: +886-2-2365-7624; fax: +886-22365-2637.

E-mail address: ccy@cc.ee.ntu.edu.tw (C.C. Yang).
}

agnosing various tissue abnormalities either on skin or inside-organ through endoscopes. OCT or ODT uses the technique of low-coherence interferometry. Typically, Michelson interferometers are built for implementing OCT scanning. Because of the longitudinal resolution in OCT scanning, which is equal to the full-width-at-half-maximum (FWHM) of interference fringe envelope, is inversely proportional to the light source bandwidth, pursuing means for generating stable broadband light source, either coherent or 
incoherent, is crucially important for OCT development. In the past, most OCT systems utilized super-luminescence light-emitting diodes as sources. Such a light source, either centered at 850 or $1300 \mathrm{~nm}$, typically has a spectral width about 30 $\mathrm{nm}$ and pig-tailed output power less than $1 \mathrm{~mW}$. This spectral width could lead to only around 15 or $35 \mu \mathrm{m}$ in depth resolution in tissues. Besides, the low available power resulted in relatively low sensitivity. The combination of two super-luminescence diodes of different center wavelengths was also used for broadening the effective spectral width and hence higher resolution [3]. Recently, based on a broadband semiconductor source (model BBS1310EB1P of AFC, Hull, Quebec, Canada) of $1310 \mathrm{~nm}$ in center wavelength, $80 \mathrm{~nm}$ in spectral width, and several $\mathrm{mW}$ in power, a depth resolution around $14 \mu \mathrm{m}$ has been reported [6]. However, this company has terminated the delivery of the aforementioned broadband source. Portable semiconductor broadband sources with even larger spectral widths and higher output powers deserve further developments. On the other hand, with ultrashort Ti:sapphire laser pulses (around $5 \mathrm{fsec}$ ), the corresponding $350 \mathrm{~nm}$ spectral width resulted in a depth resolution as small as $1.2 \mu \mathrm{m}$ [7]. Also, high laser output power can always provide sensitivity higher than $100 \mathrm{~dB}$. A broadband solid-state laser source has been used for spectral OCT, i.e., for monitoring the different response of human tissues to different wavelengths [8]. Although a 5 fsec Ti:sapphire laser can be built with efforts, such a system requires skillful attention and is not suitable for people not specially trained. To achieve deeper penetration, a mode-locked $\mathrm{Cr}$ :forsterite laser with center wavelength at $1280 \mathrm{~nm}$, was used to achieve $6 \mu \mathrm{m}$ longitudinal resolution in tissues [9]. In this work, the 50 -nm spectral width from the laser was broadened to around $150 \mathrm{~nm}$ after pulse propagation in a dispersion-shifted fiber. The spectral broadening was attributed to self-phase modulation, which interplayed with normal group-velocity dispersion to achieve a broad and Gaussianlike spectral distribution. With anomalous group-velocity dispersion, spectral broadening due to self-phase modulation can be compensated by the dispersion effect.
In this paper, we report the use of spectral broadening of femtosecond Ti:sapphire laser, based on nonlinear optics in a single-mode fiber, which is a Kerr medium [10], for broadband source of an OCT system. Widely observed nonlinear effects in the normal group-velocity dispersion range of a single-mode fiber include self-phase modulation, Raman scattering, Brillouin scattering, and four-wave mixing. With the Kerr effect in fiber, the refractive index increases linearly with optical intensity such that nonlinear phase modulation or chirp is generated for the pulsed signal. Through this self-phase modulation process, the spectrum of a short pulse is broadened. Besides self-phase modulation, stimulated Raman scattering and four-wave mixing in fiber can broaden the spectrum. Particularly, stimulated Raman scattering typically results in red shift of signal spectrum and leads to spectral asymmetry [11]. Recently, by tapering fiber [12] or using a micro-structured fiber [13], extremely broadband generation with output wavelength ranging from visible to near infrared has been reported. Based on the $100 \mathrm{fsec}$ Ti:sapphire laser, our experiments have demonstrated more than five times spectral width increase through the nonlinear optics effects in an optical fiber. As Ti:sapphire lasers of pulse widths ranging from 10 to $100 \mathrm{fsec}$ (bandwidths of $50-100 \mathrm{~nm}$ ) become popular, the proposed approach can be applied for generating even broader bandwidths and should be quite useful for many laboratories.

We used the built OCT system for scanning a human gingival mucosa sample. This sample was identified to have cancerous cells with histology. Cavity-structure abnormalities were observed in OCT scan results. Normal gingival mucosa is composed of epithelium with keratinization and underlying connective tissue. In case of malignant transformation, the epithelial cells became cancer cells and invaded into the connective tissue. Cancer cells formed nests, which were scattered in the connective tissue. Compared the histological picture with the OCT image, cancer nests correspond to the cavity structures of the OCT image.

In Section 2 of this paper, experimental procedures and spectral broadening results are described. The OCT scan results are presented in Section 3. Conclusions are drawn in Section 4. 


\section{Experimental procedures}

As shown in Fig. 1, about 100 fsec pulses from a mode-locked Ti:sapphire laser, pumped with the second-harmonics of a diode-pumped $\mathrm{Nd}: \mathrm{YVO}_{4}$ laser, were end-coupled into a single-mode fiber (3M, FS-SN-4224) with mode field (cladding) diameter of 5.18 (125) $\mu \mathrm{m}$ of about $5 \mathrm{~m}$ in length. We used such a single-mode fiber (the second-order mode cutoff at $757 \mathrm{~nm}$ ) to avoid multi-mode propagation. With multi-mode propagation, the nonlinear effects will be reduced and the output spectral width will be decreased. An isolator was used for the Ti:sapphire laser output beam before coupling into the optical fiber to avoid reflection back to the laser cavity. The pulsed signal from the fiber output end was directed to a free-space OCT system. Fig. 2 shows the input (dotted curve) and output spectra of the fiber with the input power at $300 \mathrm{~mW}$ (dashed curve) and $600 \mathrm{~mW}$ (solid curve) when the center wavelength was $828 \mathrm{~nm}$. The spectrum was asymmetrically broadened. The asymmetric output spectra may result from several possible mechanisms, including asymmetric input pulses, significant stimulated Raman scattering, and four-wave mixing. Because the center wavelength has been red-shifted from $828 \mathrm{~nm}$ to around $840 \mathrm{~nm}$, it is supposed that stimulated Raman scattering plays an important role here. In the output spectrum with input power higher than 450 $\mathrm{mW}$, a side-bump feature was observed on the short-wavelength side. The side-bump effectively increases the spectral width and may reduce the width of interference fringe envelope at the ex-

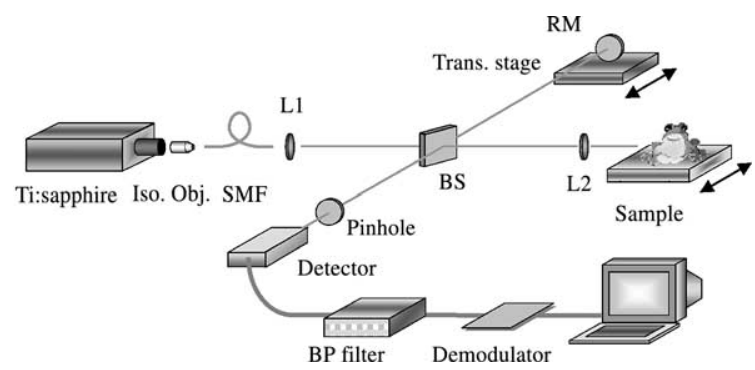

Fig. 1. Layout of the OCT system with a single-mode fiber for providing broadband source. Iso., isolator; Obj., objective; SMF, single-mode fiber; L1 and L2, lenses; BS, beam splitter; $\mathrm{RM}$, reflecting mirror.

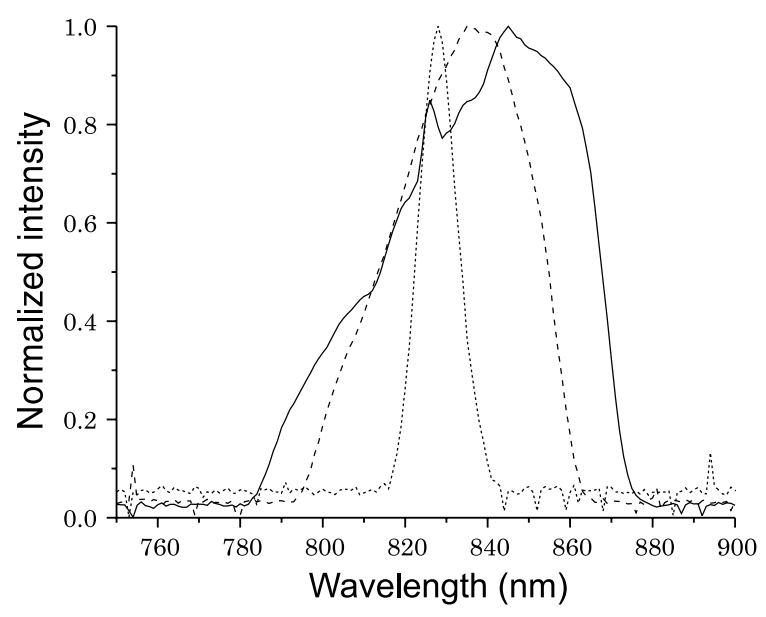

Fig. 2. Input spectrum (dotted curve) and output spectra of the fiber with the input power at $300 \mathrm{~mW}$ (dashed curve) and 600 $\mathrm{mW}$ (solid curve) when the center wavelength was $828 \mathrm{~nm}$.

pense of side-lobe generation. If the side-lobe effects can be removed with a certain image retrieval algorithm, the OCT resolution can be improved. Fig. 3 shows the dependence of output spectral width on input power with the 5-m fiber. In this figure, the circular symbols describe the FWHM spectral width versus input power. From Fig. 3, one can see the saturation trend of bandwidth increase in increasing input power. About $54 \mathrm{~nm}$ FWHM was obtained when the input power

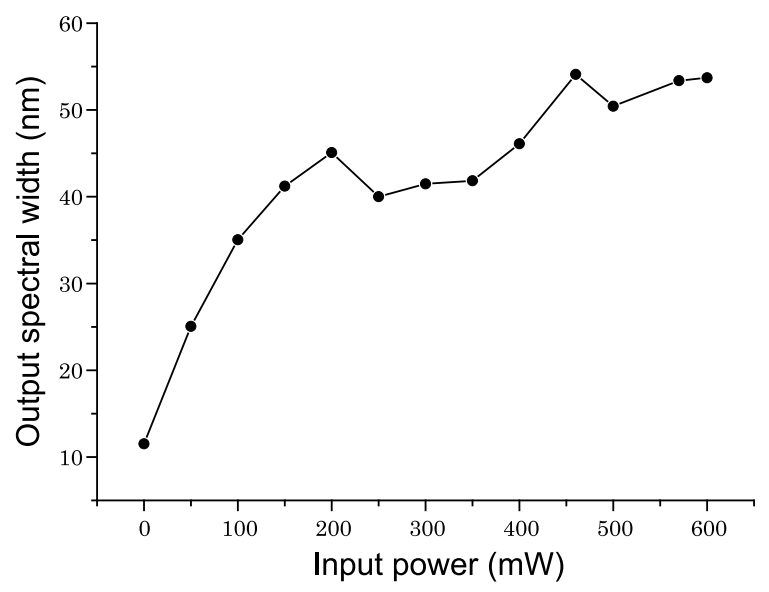

Fig. 3. Dependence of output spectral width on input power. The circular symbols describe the FWHM spectral widths of various input powers. 
reached $600 \mathrm{~mW}$. Note that because the used isolator before the fiber slightly caused pulse broadening (due to the group-velocity dispersion), the spectral broadening effect in the fiber might not be as strong as expected. Because of the limited spectral range $(815-865 \mathrm{~nm})$ of the used isolator at the laser output, our system with fiber output power higher than $400 \mathrm{~mW}$ was not stable enough for OCT operation. Hence, we chose the condition of $400 \mathrm{~mW}$ fiber output power for our OCT operation. The limited spectral range of the used isolator also explains the unsmooth output spectral width variation in Fig. 3 when the input power is larger than $200 \mathrm{~mW}$.

In the OCT system (see Fig. 1), a constantspeed stage with speed at $1 \mathrm{~mm} / \mathrm{sec}$ was used in the reference arm for phase modulation and depth scan. Fig. 4 shows the interference fringe pattern before the bandpass filter (part (a)) and its envelope after bandpass filter and demodulator (part (b)) when the input power to fiber is $400 \mathrm{~mW}$ and the output FWHM spectral width is $46 \mathrm{~nm}$ with the center wavelength shifted to around $840 \mathrm{~nm}$. It was obtained by using a glass surface for sample reflection. Because the spectrum is non-Gaussian, it causes certain side-lobes in the autocorrelation function. By proper control of the group-velocity

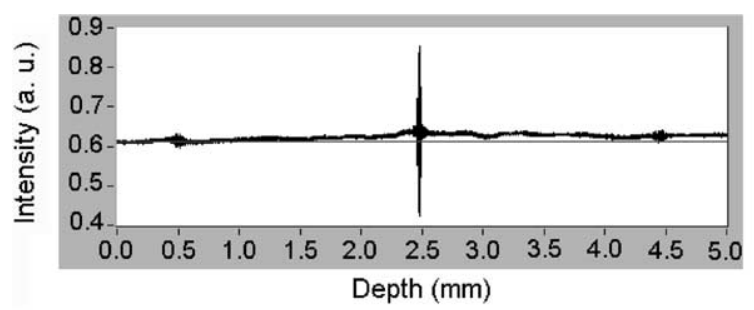

(a)

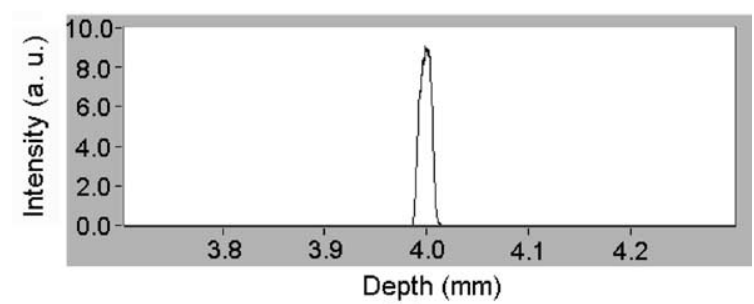

(b)

Fig. 4. (a) Interference fringe pattern and (b) its envelope of the OCT system when the input power to the fiber is $400 \mathrm{~mW}$ and the output spectral width is $46 \mathrm{~nm}$. dispersion in the reference arm, the side-lobes can be suppressed. The FWHM of this envelope is 12 $\mu \mathrm{m}$, corresponding to $8.6-\mu \mathrm{m}$ longitudinal resolution of the OCT system in tissues if the index of refraction for the tissue was assumed to be 1.4. Fig. 4(a) also shows two satellite features about $2 \mathrm{~mm}$ away from the main feature. They might originate from the effects of multi-reflection in the OCT system. They set a limitation of the scanning depth as $2 \mathrm{~mm}$. Nevertheless, $2 \mathrm{~mm}$ is large enough for biomedical applications. The non-zero background in Fig. 4(a) may limit the dynamic range of the interferometry measurement. However, it can be reduced with a bandpass filter in data processing. A lens with $3 \mathrm{~mm}$ in focal length was used for focusing light beam onto samples. The beam size at the focal point was estimated to be $5 \mu \mathrm{m}$, which corresponded to the lateral resolution of OCT scanning. The focal spot size estimation was obtained from the beam diameter incident onto the focusing lens. The high lateral resolution was chosen at the expense of a short depth of focus or depth range of scanning, which was estimated to be just a few hundreds $\mu \mathrm{m}$. The available power for sample illumination could be as high as 100 $\mathrm{mW}$. However, we used only $20 \mathrm{~mW}$ for sample illumination, which is believed to be under the safety level. A pinhole was used before the photodetector for spatially filtering stray light such that the sensitivity of the system could be improved. With insertion of calibrated neutral density filters of $50 \mathrm{~dB}$ attenuation in both reference and sample arms under the condition that $>3 \mathrm{~dB}$ signalto-noise ratio was maintained, the sensitivity was estimated to be higher than $100 \mathrm{~dB}$.

\section{OCT scan results}

We used the built OCT system to scan various tissue samples. With the condition of $46 \mathrm{~nm}$ FWHM spectral width, Fig. 5(a) shows the OCT scanning result of a piece of human gingival mucosa, which belonged to a patient having oral cancer. The fresh sample was first scanned with the OCT system and then preserved in formalin for histological imaging. Cavity structures (as a few examples indicated with arrows) in the left portion, 


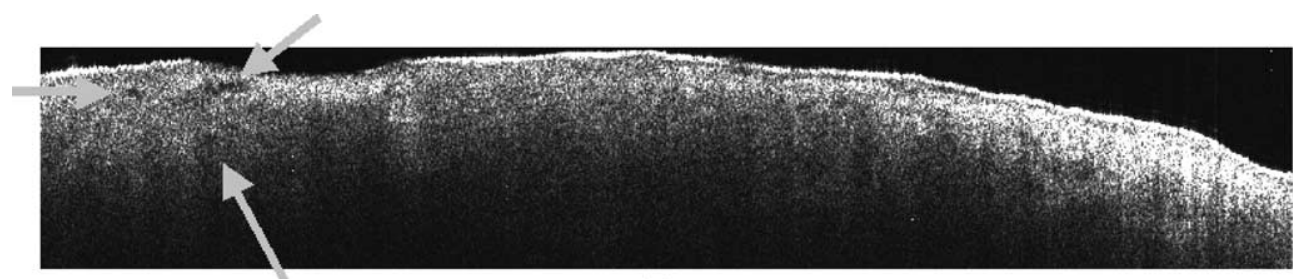

(a)

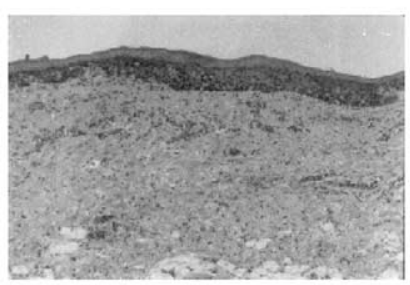

(b)

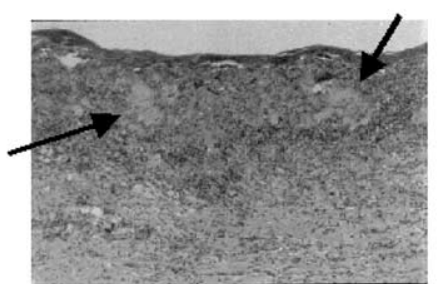

(c)

$1 \mathrm{~mm}$

Fig. 5. (a) An OCT scan image of a human gingival mucosa sample. In the left portion, cavity structures, as indicated with arrows, can be clearly seen. (b) A histological image of a normal portion of the sample. (c) A histological image of the cancerous portion of the sample showing the cancer nests (as indicated with arrows). The same scale is used for the three images.

including some of them close to the surface, show a significant contrast against normal tissues at the right end of the picture. For comparison, Figs. 5(b) and (c) show the histological images of a normal portion and a malignant portion of this sample, respectively. In Fig. 5(b), the connective tissue can be clearly distinguished in the normal portion, while in Fig. 5(c) the epithelial cells became cancer cells and invaded into the connective tissues, which disappeared. Nests were formed with cancer cells. The regions of darker contrast (as a few examples indicated with arrows) in Fig. 5(a) represent cancer nests. The cavity structures (regions of darker contrast) observed in the OCT scan are supposed to correspond to the cancer nests in the sample.

\section{Conclusions}

In summary, we have built an OCT system with its broadband source generated with nonlinear optics processes, at least including self-phase modulation and Raman scattering, in a singlemode fiber. The mechanisms of spectral broadening can be further investigated with numerical simulations of laser pulse propagation in optical fiber under the influences of various nonlinear effects as well as group-velocity dispersion. With 400 $\mathrm{mW}$ power coupled into the fiber, an FWHM bandwidth of $46 \mathrm{~nm}$ was obtained. Such a broadband source corresponded to an OCT depth resolution of $8.6 \mu \mathrm{m}$ in tissues. The bandwidth can be further increased with a tapered fiber [12] or a micro-structured fiber [13]. The OCT system was used for scanning a cancerous human gingival mucosa sample. Cavity-structure abnormalities, which corresponded to cancer nests in histological pictures, were clearly observed. Further study of oral cancer based on the OCT technique is under way. For clinical applications, OCT systems with compact broadband light sources are preferred. However, before such compact broadband light sources can be improved and become commercially available, our proposed method should be useful for further development of OCT.

\section{Acknowledgements}

This research was supported by National Health Research Institute, The Republic of China, under the grant of NHRI-GT-EX89E819L. 


\section{References}

[1] D. Huang, E.A. Swanson, C.P. Lin, J.S. Schuman, W.G. Stinson, W. Chang, M.R. Hee, T. Flotte, K. Gregory, C.A. Puliafito, J.G. Fugimoto, Science 254 (1991) 1178.

[2] J.F. de Boer, S.M. Srinivas, B.H. Park, T.H. Pham, Z. Chen, T.E. Milner, J.S. Nelson, IEEE J. Sel. Top. Quantum Electron. 5 (1999) 1200.

[3] J.M. Schmitt, IEEE J. Sel. Top. Quantum Electron. 5 (1999) 1205.

[4] Y. Zhao, Z. Chen, C. Saxer, S. Xiang, J.F. de Boer, J.S. Nelson, Opt. Lett. 25 (2000) 114.

[5] S. Yazdanfar, A.M. Rollins, J.A. Izatt, Opt. Lett. 25 (2000) 1448.

[6] C.E. Saxer, J.F. de Boer, B.H. Park, Y. Zhao, Z. Chen, J.S. Nelson, Opt. Lett. 25 (2000) 1355.
[7] W. Drexler, U. Morgner, F.X. Kärtner, C. Pitris, S.A. Boppart, X.D. Li, E.P. Ippen, J.G. Fujimoto, Opt. Lett. 24 (1999) 1221.

[8] U. Morgner, W. Drexler, F.X. Kärtner, X.D. Li, C. Pitris, E.P. Ippen, J.G. Fujimoto, Opt. Lett. 25 (2000) 111.

[9] B.E. Bouma, G.J. Tearney, I.P. Bilinsky, B. Golubovic, J.G. Fujimoto, Opt. Lett. 21 (1996) 1839.

[10] G.P. Agrawal, Nonlinear Fiber Optics, second ed., Academic, San Diego, California, 1995.

[11] F.M. Mitschke, L.F. Mollenauer, Opt. Lett. 11 (1986) 659.

[12] T.A. Birks, W.J. Wadsworth, P.St.J. Russel, Opt. Lett. 25 (2000) 1415.

[13] A. Ferrando, E. Silvestre, J.J. Miret, P. Andres, Opt. Lett. 25 (2000) 790. 